\title{
MS070.P01
}

\section{Crystal structure of the new superconductor FeSe1-xSx}

Kirill V. Frolov ${ }^{1}$, Anna G. Ivanova ${ }^{1}$, Dmitry Yu. Chernishov ${ }^{2}$, Iurii Dovgaliuk ${ }^{2}$, Dmitry A. Chareeev ${ }^{3}$, Ivan A. Troyan ${ }^{1}$

${ }^{1}$ FSRC Crystallography And Photonics RAS, Moscow, Russian Federation, ${ }^{2}$ Swiss-Norwegian Beamlines at the ESRF, Grenoble, France, ${ }^{3}$ Institute of Experimental Mineralogy RAS, Chernogolovka, Moscow Region, Russian Federation E-mail: green@crys.ras.ru

The discovery of superconductivity in iron-based pnictides and chalcogenides has been at the forefront of interest over the last few years $[1,2]$. $\mathrm{Fe}(\mathrm{Se}, \mathrm{Te}, \mathrm{S})$ compounds considered as the simplest Fe-based superconductors useful for study correlations between structural, electron, magnetic and superconducting properties. Among these materials FeSe1-xSx is the least studied compound and single crystal X-ray diffraction (XRD) experiments for it was not conducted.

FeSe1-xSx $(x=0-0.2)$ single crystals were grown in evacuated quartz ampoules using the $\mathrm{AlCl} / 3 / \mathrm{KCl}$ flux technique [3] in a temperature gradient (from $400^{\circ} \mathrm{C}$ to $\sim 50^{\circ} \mathrm{C}$ ) for 45 days. Crystals have a platelike shape with the $\mathrm{C}$ axis oriented perpendicular to the crystal plane. Two samples with $x=0.03$ and 0.09 were selected for single crystal synchrotron XRD measurements. Both of them were superconducting with Tc $=9.5$ and $10.1 \mathrm{~K}$ respectively. The XRD data were collected in 90 - $300 \mathrm{~K}$ temperature range at the ESRF beamline BM01 using PILATUS@SNBL diffractometer $(\lambda=0.7458 \AA$, PILATUS2M detector) equipped with Oxford Cryojet cryogenic nitrogen jet system.

Complete single crystal XRD measurements were performed for good quality FeSe0.91S0.09 sample at the room temperature. Crystal structure of FeSe0.91S0.09 was refined in sp.gr. P4/n ( $a=3.809(1), c=5.529(1), R=3.5 \%)$. It was found that atoms of $\mathrm{S}$ and Se statistically occupy 2c position of the structure. Percentage ratio $\mathrm{S} / \mathrm{Se}$ was defined in the result of the site occupancies refinement. Test XRD room temperature experement for FeSe0.97S0.03 crystal showed that sample was polycrystalline. Polycrystalline low temperature XRD measurements were performed in temperature interval $90-$ $300 \mathrm{~K}$. Some additional peaks not corresponding to sp.gr. P4/n were revealed at temperatures below $170 \mathrm{~K}$.

The reported study was funded by Ministry of Education and Science of the Russian Federation (Project \#14.616.21.0068).

[1] Kamihara Y. et al.(2008). J. Am. Chem. Soc. 130, 3296-3297.

[2] Hsu F.C. et al. (2008). Proc. Natl. Acad. Sci. U.S.A. 105 14262-14264.

[3] Chareev D. et al. (2013). Cryst. Eng. Commun. 15, 1989-1993.

Keywords: iron-based superconductors, single crystal structure, synchrotron radiation 